\title{
Role of Sentinel Lymph Node Biopsy in Papillary Thyroid Carcinoma in Vietnam
}

\author{
Le Van QUANG, Nguyen Van HIEU, Nguyen Xuan HAU, Nguyen Van HUNG \\ Hanoi Medical University, Department of Oncology, Hanoi, VIETNAM
}

\begin{abstract}
This research will provide the feasible, predictive values in metastasis of SLN biopsy among Vietnamese patients with thyroid carcinoma. 122 patients were recruited in this study with 18 men (14.8\%) and 104 women (85.2\%). Tumors were in T1 stage in 59 patients (48.4\%), in T2 stage in 14 patients (11.5\%), in T3 stage in 45 patients (36.9\%), and in T4 stage in 4 patients (3.2\%). The SLN was identified by intraoperative injection of methylene blue in all cases (100\%). Metastasis in SLN was reported in 53 cases (43.4\%). Sensitivity, specificity, accuracy, positive and negative predictive values of SLN biopsy were $79.1 \%, 100 \%, 87.7 \%, 100 \%$ and $79.7 \%$, respectively. SLN biopsy is technically feasible and safe procedure, which can reveal occult metastasis among PTC patients with cNO stage, who may get benefit from selective neck dissection. However, blue method of SLN detection makes SLN biopsy of limited use in the control of clinically node-negative papillary thyroid carcinoma because of low sensitivity and a high false-negative rate.
\end{abstract}

Keywords: Sentinel lymph node biopsy, Methylene blue; Metastasis, Papillary thyroid cancer, Selective neck dissection

\section{INTRODUCTION}

Papillary thyroid cancer (PTC) is the most common type of differentiated thyroid cancer and spreads mainly via the lymphatic system. ${ }^{1}$ Microscopic metastases were revealed in approximately $80 \%$ of adult patients with PTC. ${ }^{2}$ It is estimated that $27-90 \%$ of PTC cases with occult metastasis of lymph nodes are detected after surgery and histologic examination..$^{3-4}$ Although thyroidectomy with lymphadenectomy significantly increases the rate of postoperative complications, especially hypocalcemia ${ }^{5-7}$, recurrence appears mostly in lymph node and node metastasis can only be treated by surgery. ${ }^{8-12}$ In addition, some studies show that a second surgery after recurrence of the central nodes metastasis increases the risk of complications such as recurrent nerve paralysis and parathyroid dysfunction. ${ }^{13-14}$ As a result, finding a method to detect occult lymph node metastasis in thyroid carcinoma is necessary for planning of treatment. Sentinel lymph nodes (SLNs) are defined as the first lymph nodes in the lymphatic drainage area, which receive lymph flow from primary tumors and are supposed to reflect the status of the remaining lymph nodes. ${ }^{15}$ The result from SLN biopsy can be used to decide whether to perform lymphadenectomy and for pathology staging. ${ }^{16-18}$ Advantage of SLN biopsy is that it can detect regional metastatic lymph nodes smaller than $2 \sim 3 \mathrm{~mm}$ in the greatest dimension that is impossible to do by other technics such as highresolution ultrasonography. 
Moreover, in contrast to metastases in the lateral neck, which can be detected by ultrasonography with high sensitivity and specificity ${ }^{18}$ metastases in the central neck compartment are hidden by the thyroid gland, multifocal nodules, clavicle and sternum, that cause low sensitivity of ultrasonography. ${ }^{18}$ Recently, SLN biopsy has become a common method for diagnostic of several types of cancer, especially melanoma and breast cancer. ${ }^{19-20}$ Benefits of SLN biopsy in breast cancer are clear: it lets to reduce the need for axillary lymph node dissection, which can cause edema of arms. ${ }^{21}$ However, SLN biopsy application to the treatment of PTC patients still has not been thoroughly investigated. This research will provide the feasible, predictive values in metastasis of SLN biopsy among Vietnamese patients with PTC.

\section{PATIENTS AND METHODS}

\section{Patients}

This prospective study included 112 patients with PTC who underwent SLN biopsy at Oncology and Palliative care Department of Hanoi Medical University Hospital during 1/2015- 1/2017. All patients were diagnosed with PTC by preoperative fine-needle aspiration cytology and had no preoperative evidence of cervical lymph node metastasis at physical and ultrasound examination. Patients with palpable or ultrasound detected lymph node involvement, distant metastasis, previous history of thyroid surgery, and other types of thyroid malignancies were excluded.

\section{Surgical Procedure and Detection of SLN}

In this study, all patients underwent bilateral level VI dissection. A transverse low-collar skin incision was followed by separation of the skin flap and a longitudinal incision at the linea alba cervicalis. Then, the thyroid capsule was carefully opened to expose the thyroid gland completely without injury of the capsule. The thyroid gland and the ipsilateral jugular vein were exposed. Then, approximately $1 \mathrm{ml}$ of methylene blue was injected into 4 sites (upper, lower, lateral and central) surrounding the primary tumor. Within minutes, the stained lymphatic vessels from the primary tumor became apparent, and blue-stained lymph nodes were iden- tified via tracing the stained lymphatic vessels. These blue-stained lymph nodes, defined as SLN, were carefully collected and sent to the Pathology Department of Hanoi Medical University Hospital for frozen section and definitive pathology investigations. Subsequently, total thyroidectomy or lobectomy + isthmusectomy were performed. All patients also underwent bilateral selective neck lymphadenectomy, defined as non-SLN. Selective lymph node dissections included bilateral level VI dissection; level II, III and IV dissection in both sides. Samples from thyroid gland and neck lymph nodes were sent for pathological examination and data were analyzed to evaluate clinical values of SLN biopsy.

\section{Statistical Analysis}

All data from research record and pathological results were imported into EpiData software and were analyzed in RStudio 1.0.136 software. The accuracy of the results on the correlation between lymph node metastasis and age, sex, tumor characteristics, as well as between SLN metastasis and the same indicators was confirmed using Pearson's Chi-squared test with Yates' continuity correction.

\section{RESULTS}

The characteristics of all subjects are shown in Table 1. There were 122 patients, who underwent SLN biopsy, among which female/male ratio was 5.8. The mean age was 42.4 years (ranging from 20 to 68 years). More than half of the patients had tumor size below $1 \mathrm{~cm}(54.9 \%)$. The majority of the patients had unifocal lesion $(82 \%)$ and normal TSH level (88.5\%). According to neck ultrasound results, most patients $(73.8 \%)$ had nodules TIRADS 4 (Thyroid Imaging Reporting And Data System). ${ }^{22}$ TIRADS 4 refers to suspicious nodules (5-80\% malignancy rate). ${ }^{22}$ During surgery, $\mathrm{T}$ stage was evaluated. Among 122 patients, 59 patients $(48.4 \%)$ had $\mathrm{T} 1$ stage, 14 patients $(11.5 \%)$ had T2 stage, 45 patients $(36.9 \%)$ had T3 stage and 4 patients (3.2\%) had T4 stage of tumor.

Sentinel lymph nodes were successfully detected in all cases (Table 2). The average number of detected SLN was 4. SLN located mainly at the lev- 


\begin{tabular}{|c|c|c|}
\hline Characteristic & Value & $\begin{array}{l}\text { Percentage } \\
\text { (\%) }\end{array}$ \\
\hline \multicolumn{3}{|l|}{ Age } \\
\hline$<45$ & 76 & 62.3 \\
\hline$>45$ & 46 & 37.7 \\
\hline Mean \pm SD (range) & \multicolumn{2}{|c|}{$42.39 \pm 11.32(20-68)$} \\
\hline \multicolumn{3}{|l|}{ Sex } \\
\hline Male & 18 & 14.8 \\
\hline Female & 104 & 85.2 \\
\hline \multicolumn{3}{|l|}{ Tumor size } \\
\hline$\leq 1 \mathrm{~cm}$ & 67 & 54.9 \\
\hline$>1 \mathrm{~cm}$ & 55 & 45.1 \\
\hline \multicolumn{3}{|l|}{ Multifocality } \\
\hline Multifocal & 22 & 18.0 \\
\hline Unifocal & 100 & 82.0 \\
\hline \multicolumn{3}{|l|}{$\mathrm{TSH}$} \\
\hline Abnormal & 14 & 11.5 \\
\hline Normal & 108 & 88.5 \\
\hline \multicolumn{3}{|l|}{ Position of tumor } \\
\hline Right lobe & 66 & 54.1 \\
\hline Left lobe & 49 & 40.2 \\
\hline Isthmus & 3 & 2.5 \\
\hline Both lobes & 4 & 3.3 \\
\hline \multicolumn{3}{|l|}{ TIRADs } \\
\hline 3 & 22 & 18.0 \\
\hline 4 & 90 & 73.8 \\
\hline 5 & 10 & 8.2 \\
\hline \multicolumn{3}{|l|}{ T stage } \\
\hline $\mathrm{T} 1$ & 59 & 48.4 \\
\hline T2 & 14 & 11.5 \\
\hline T3 & 45 & 36.9 \\
\hline $\mathrm{T} 4$ & 4 & 3.2 \\
\hline
\end{tabular}

el VI (86.9\%), followed by level VI + Ipsilateral jugular chain $(9.8 \%)$ and ipsilateral jugular chain $(3.3 \%)$, while at the contralateral jugular chain no SLN were detected. After resection, SLN biopsies were sent to prepare frozen section and the results were compared with specimen of routine selective neck lymph node dissection, which were sent for final pathology investigations. The positive rate of SLN lesion in frozen section and final pathology investigations was $43.4 \%$ and $46.7 \%$ respectively. There were 4 cases with negative results in frozen section analysis but positive results in permanent

\begin{tabular}{|ll|}
\hline \multicolumn{2}{|l|}{ Table. Sentinel lymph node detection } \\
\hline Number of patients with detected SLN & $122(100 \%)$ \\
Average number of SLN & $4.0(1-12)$ \\
Position & \\
$\quad$ Level VI & $106(86.9 \%)$ \\
$\quad$ Level VI + Ipsilateral jugular chain & $12(9.8 \%)$ \\
Ipsilateral jugular chain & $4(3.3 \%)$ \\
Contralateral jugular chain & $0(0 \%)$ \\
Positive SLN & \\
Frozen section & $53(43.4 \%)$ \\
Permanent pathology & $57(46.7 \%)$ \\
\hline
\end{tabular}

pathology. Pathology results of the selective lymph node dissection are represented in the Table 3. Level VI lymph node had highest metastasis rate (45.1\%) while the occult metastasis rate was only $5.7 \%$ in contralateral neck lymph node group and $22.1 \%$ in the ipsilateral neck lymph node. Sensitivity, specificity, accuracy, positive and negative predictive values of SLN biopsy were 79.1\%, 100\%, $87.7 \%, 100 \%$ and $79.7 \%$, respectively (Table 4 ).

Male had higher risk of neck lymph node metastasis compared to female $(83.3 \%$ vs. $50 \%$, p= 0.018 ), while patients $\leq 45$ years old had higher risk of SLN metastasis compared to younger patients. However, other factor such as T stage, tumor size, multifocality and TSH level were not significantly related to both neck lymph node metastasis and SLN metastasis (Table 5 and 6).

\section{DISCUSSION}

When we are talking about papillary thyroid cancer, lymph node metastasis is related to locoregional recurrence. ${ }^{11}$ Neck lymph node resection can reduce risk of recurrence, however it can also increase the rate of postoperative complications. ${ }^{5-7}$ Therefore, estimation of the benefit of reducing recurrent rate and the risk of increasing complications in treatment of PTC is still on debate. If metastasis in lymph nodes cannot be confirmed before surgery, SLN biopsy can be used to decide whether to perform lymphadenectomy. ${ }^{16-18}$ Recently, SLN biopsy has become a potential minimally invasive surgery procedure to detect nodes in patients with 
International Journal of Hematology and Oncology

\begin{tabular}{|c|c|c|c|}
\hline Neck lymph node status & $\begin{array}{l}\text { Ipsilateral level II, III and } \\
\text { IV neck lymph node }\end{array}$ & $\begin{array}{l}\text { Contralateral level II, III and } \\
\text { IV neck lymph node }\end{array}$ & Level VI lymph node \\
\hline Negative & 95 (77.9\%) & 115 (94.3\%) & 67 (54.9\%) \\
\hline Positive & $27(22.1 \%)$ & $7(5.7 \%)$ & 55 (45.1\%) \\
\hline Total & 122 & 122 & 122 \\
\hline
\end{tabular}

\begin{tabular}{|ll|}
\hline Table 4. Parameters of SLN biopsy \\
\hline Parameters & Value \\
\hline Sensitivity & $79.1 \%$ \\
Specificity & $100 \%$ \\
Accuracy & $87.7 \%$ \\
Positive predictive value & $100 \%$ \\
Negative predictive vale & $79.7 \%$ \\
\hline
\end{tabular}

melanoma, breast cancer and thyroid cancer. ${ }^{20-21}$ The main advantage of SLNB is to identify patients with negative lymph node to avoid unnecessary procedure and risk from surgery. ${ }^{23}$

For description of the location and the anatomic boundaries of lymph node groups in the neck, a lot of classifications were suggested, based on computed tomography and magnetic resonance imaging, surgical landmarks or physical assessment criteria. ${ }^{24}$ American Joint Committee on Cancer (AJCC) and the American Academy of Otolaryngology-Head and Neck Surgery (AAO-HNS) revealed the most commonly used among them. ${ }^{25}$ The level system, that unites certain lymph nodes, which are located within different levels of the neck, is used in these classifications. ${ }^{26}$ There are five levels of neck division on each side, while for description of lymph nodes in the anterior neck the sixth level is used. ${ }^{27}$ Level VI is defined as anterior (central) neck compartment and includes pretracheal and paratracheal nodes, the precricoid (Delphian) node, and the perithyroidal nodes. PTC can be spread through these nodes and connecting lymphatic channels, that rises recommendation for them to be delated during surgery of thyroid cancer. During current investigation, it was shown that lymph nodes situated at the Level VI had the highest metastasis rate

(45.1\%). It is consistent with evaluable data, which pointed that lesions in the level VI were found in up to $90 \%$ of node positive $(\mathrm{N}+)$ patients, while the frequency of lateral node involvement among the patients with positive nodes may reach $51-100 \%$, where the fraction of contralateral node is $18.4 \%$ in patients with uni-nodular thyroid tumor. ${ }^{27}$

Many methods of SLN identification has been published. ${ }^{21,28-32}$ Kelemen et al was the first who stated the concept of SLN in thyroid carcinoma by using patent blue dye ${ }^{28}$; Fukui et al. analyzed the SLN biopsy using methylene blue staining in patients with PTC and confirmed that it is safe and feasible technique. ${ }^{29}$ Catarci and colleagues performed a combined technique of SLN mapping using patent blue $\mathrm{V}$, preoperative $99 \mathrm{mTc}$ and intraoperative hand-held gamma probe and insisted on using of the combination of nuclear medicine imaging and lymphatic vital dye to get the most accurate results of sentinel node identification. ${ }^{30}$ Jee Soo Kim et al. showed that methylene blue has higher sensitivity and lower overall false negative rates compared to radioactive tracer using, but overall sentinel node identification rates for vital blue dye alone was determined to be lower than those of radioisotope alone..$^{21}$ It is true that radioisotope, and combined techniques especially (radioisotope + blue dye), give better results than blue dye alone, and so they are widely used, but these procedures are expensive, and require use in radiological laboratory with special equipment. The latter fact may significantly limit the use of radioisotopes in sentinel node identification (method may not be available in some clinics and/or for some patients) and make the use of blue dye alone more cost-efficient, and in some cases, more practical and preferable. Besides, experienced surgeons may success in identifying sentinel nodes using blue dye alone. Aside 


\begin{tabular}{|c|c|c|c|}
\hline \multirow[t]{2}{*}{ Factor } & \multicolumn{2}{|c|}{$\begin{array}{l}\text { Neck lymph node } \\
\text { metastasis }\end{array}$} & \multirow[t]{2}{*}{$p$-value } \\
\hline & Yes & No & \\
\hline \multicolumn{4}{|l|}{ Age } \\
\hline$\leq 45$ & 46 & 30 & \multirow[t]{2}{*}{0.158} \\
\hline$>45$ & 21 & 25 & \\
\hline \multicolumn{4}{|l|}{ Sex } \\
\hline Male & 15 & 3 & \multirow[t]{2}{*}{0.018} \\
\hline Female & 52 & 52 & \\
\hline \multicolumn{4}{|l|}{ T stage } \\
\hline T1-2 & 43 & 30 & \multirow[t]{2}{*}{0.371} \\
\hline T3-4 & 24 & 25 & \\
\hline \multicolumn{4}{|l|}{ Tumor size } \\
\hline$\leq 1 \mathrm{~cm}$ & 32 & 35 & \multirow[t]{2}{*}{0.116} \\
\hline$>1 \mathrm{~cm}$ & 35 & 20 & \\
\hline \multicolumn{4}{|l|}{ Multifocality } \\
\hline Multifocal & 9 & 13 & \multirow[t]{2}{*}{0.222} \\
\hline Unifocal & 58 & 42 & \\
\hline \multicolumn{4}{|l|}{ TSH } \\
\hline Abnormal & 6 & 8 & \multirow[t]{2}{*}{0.498} \\
\hline Normal & 61 & 47 & \\
\hline
\end{tabular}

from that, radioisotopes are associated with radiation exposure of personnel that contacts with a patient. In the context of Vietnam, blue dye method seems to be an adequate method that can be applied to detect SLN, as it requires little time, effort or expenses, not mentioning that this method is still in use. Although various blue dyes can be used (such as methylen blue, isosulfan blue or patent blue V), universal dye does not exist; dyes have their own side effects, and data on their effectiveness are contradictory and require further study. This research is not about the effectiveness and safety of different blue dyes, but methylene blue appears to be safer because of a rare adverse reactions report. ${ }^{31}$ Besides, it has been shown to be equally effective. Based on theses facts, methylene blue dye was selected for this research.

In this study, the SLN was detected by intraoperative injection of methylene blue in all cases (100\%) with the average number of SLN, equal 4. During comparing of SLN frozen section results and neck lymph node final pathology results, this study

\begin{tabular}{|c|c|c|c|}
\hline \multirow[t]{2}{*}{ Factor } & \multicolumn{2}{|c|}{ SLN metastasis } & \multirow[t]{2}{*}{ p-value } \\
\hline & Yes & No & \\
\hline \multicolumn{4}{|l|}{ Age } \\
\hline$\leq 45$ & 39 & 37 & 0.039 \\
\hline$>45$ & 14 & 32 & \\
\hline \multicolumn{4}{|l|}{ Sex } \\
\hline Male & 10 & 8 & 0.387 \\
\hline Female & 43 & 61 & \\
\hline \multicolumn{4}{|l|}{ T stage } \\
\hline T1-2 & 34 & 39 & 0.506 \\
\hline T3-4 & 19 & 30 & \\
\hline \multicolumn{4}{|l|}{ Tumor size } \\
\hline$\leq 1 \mathrm{~cm}$ & 26 & 41 & 0.339 \\
\hline$>1 \mathrm{~cm}$ & 27 & 28 & \\
\hline \multicolumn{4}{|l|}{ Multifocality } \\
\hline Multifocal & 5 & 17 & 0.054 \\
\hline Unifocal & 48 & 52 & \\
\hline \multicolumn{4}{|l|}{$\mathrm{TSH}$} \\
\hline Abnormal & 5 & 9 & 0.739 \\
\hline Normal & 48 & 60 & \\
\hline
\end{tabular}

showed that sensitivity, specificity, accuracy, positive and negative predictive values of SLN biopsy were $79.1 \%, 100 \%, 87.7 \%, 100 \%$ and $79.7 \%$, respectively. Our results were fairly similar to DanGui Yan when researching 51 patients, with indices of $97.4 \%, 100 \%, 98 \%, 100 \%$ and $92.3 \%$, respectively. ${ }^{33}$ Lower sensitivity in our study may come from the fact that the latter research used intraoperative radioisotype, thus the number and detection rate of SLN increased, as a result, sensitivity may be higher. Previous study confirmed that combination of radioisotype and blue dye methods could reduce false negative rate and increase detection rates of sentinel lymph node metastases, compared to the dye method only. ${ }^{34}$ However, by using methylene blue, we still increase the chance of finding SLN and also can rule out negative SLN cases accurately from neck dissection procedure. On the other hand, when comparing with other studies, that used methylene blue, sensitivity, specificity, positive and negative predictive values were similar to our data with $78 \%, 78.8 \%, 100 \%, 100 \%$ and 
$84.3 \%$ respectively. ${ }^{35}$ Thus, we will use combined technique in the future investigations.

Our study also found that central compartment lymph node metastasis rate was the highest (54.9\%), followed by ipsilateral level II, III and IV lymph nodes metastasis rate $(22.1 \%)$. Contralateral level II, III and IV neck lymph node metastasis rate was the lowest (5.7\%). Noguchi during research on 68 patients with selective neck lymphadenectomy, found that $78 \%$ of lymph node metastasis belonged to central compartment lymph node and $22 \%$ belonged to carotid lymph node. ${ }^{36}$ Some studies also pointed that central compartment lymph node metastasis rate varied from $27 \%$ to $90 \%$, while contralateral neck lymph node metastasis rate was low. ${ }^{1-3}$ Although some researchers have advocated routine prophylactic modified radical neck dissection $^{37}$, the benefit of modified radical neck dissection in cases of node-negative PTC is still unclear and is not recommended by current guidelines. ${ }^{38}$ Because of the low rate of occult metastasis of contralateral neck lymph node in our study, routine lymph node dissection in contralateral compartment may not be necessary except cases involved in positive node evidence.

When analysing relationship between lymph node metastasis and other factors such as age, $\mathrm{T}$ stage, sex, tumor size, multifocality, and TSH level; it was found that only sex was independent predict factor for neck lymph node metastasis and only age was independent predict factor for SLN metastasis. In previous studies, male gender was significantly associated with higher rates of neck lymph node metastasis and was an important indicator for prophylatic lateral node dissection39, but for central node metastasis sex was not predictive factor, as well as, age, tumor size, multifocality, bilaterality etc. ${ }^{40}$ Yan et al. conducted research on 212 PTC patients and found that age was not predictive factor for central compartment lymph node metastasis, while the frequency of subclinical central lymph node metastasis was slightly greater in patients aged under 45 years ${ }^{23}$ similarly to our research. It is consistent with previous data, which confirmed that the highest incidence of nodal metastasis is observed in young people (up to $80 \%$ of children), who, nevertheless, have excellent prognosis, that highlights the importance of age in the initial diagnosis. ${ }^{27}$ Other studies, however, reported that age was not associated with lymph node metastasis in PTC. ${ }^{29-31}$ Overall, in our study SLN biopsy revealed metastasis in 53 of 57 cases, confirmed by permanent pathology. This gives us the ability to use SLN biopsy to confirm the absence of metastasis in SLN and thus, to avoid unnecessary central compartment neck dissection. Benefits of SLN biopsy in identifying and excising of clinically occult metastases in PTC patients were confirmed by prior studies..$^{34,41-43}$ Moreover, since many factors could not predict accurately lymph node metastasis condition, we argue that SLN biopsy should be performed systematically in all cases of clinical node-negative papillary thyroid carcinoma.

\section{Conclusion}

Our study implys that SLN biopsy with methylene blue dye method is technically feasible and safe method, which can reveal occult metastasis with among PTC patients with $\mathrm{cNO}$ stage who may get benefit from selective neck dissection. However, blue method of SLN detection makes SLN biopsy of limited use in the control of clinically node-negative papillary thyroid carcinoma because of low sensitivity and a high false-negative rate.

\section{REFERENCES}

1. Henry JF, Gramatica L, Denizot A, et al. Morbidity of prophylactic lymph node dissection in the central neck area in patients with papillary thyroid carcinoma. Langenbecks Arch Surg 383: 167-169, 1998.

2. Shaha AP. Management of the neck in thyroid cancer. Otorhinolaryngol Clin N Am. 31: 823-831, 1998.

3. Shaha AR. Thyroid cancer: extent of thyroidectomy. Cancer Control J Moffitt Cancer Cent 7: 240-245, 2000.

4. Hay ID, Bergstralh EJ, Goellner JR, et al. Predicting outcome in papillary thyroid carcinoma: development of a reliable prognostic scoring system in a cohort of 1779 patients surgically treated at one institution during 1940 through 1989. Surgery 114: 1050-1058, 1993.

5. Roh J-L, Park J-Y, Park Cl. Prevention of postoperative hypocalcemia with routine oral calcium and vitamin D supplements in patients with differentiated papillary thyroid carcinoma undergoing total thyroidectomy plus central neck dissection. Cancer 115: 251-258, 2009. 
6. Bergenfelz A, Jansson S, Kristoffersson A, et al. Complications to thyroid surgery: results as reported in a database from a multicenter audit comprising 3,660 patients. Langenbecks Arch Surg 393: 667-673, 2008.

7. Shen WT, Ogawa L, Ruan D, et al. Central neck lymph node dissection for papillary thyroid cancer: The reliability of surgeon judgment in predicting which patients will benefit. Surgery 148: 398-403, 2010.

8. Jarzab B, Sporny S, Lange D, et al. Diagnosis and treatment of thyroid cancer - Polish guidelines. Endokrynol Pol 61: 518-568, 2010.

9. Cady B, Rossi R. An expanded view of risk-group definition in differentiated thyroid carcinoma. Surgery 104: 947953, 1988.

10. Rossi RL, Cady B, Silverman ML, et al. Current results of conservative surgery for differentiated thyroid carcinoma. World J Surg 10: 612-622, 1986.

11. Beasley NJ, Lee J, Eski S, et al. Impact of nodal metastases on prognosis in patients with well-differentiated thyroid cancer. Arch Otolaryngol Head Neck Surg 128: 825-828, 2002.

12. Weber $\mathrm{T}$, Amann $\mathrm{K}$, Weckauf $\mathrm{H}$, et al. Detection of disseminated medullary thyroid carcinoma cells in cervical lymph nodes by cytokeratin 20 reverse transcription-polymerase chain reaction. World J Surg 26: 148-152, 2002.

13. Mårtensson $\mathrm{H}$, Terins J. Recurrent laryngeal nerve palsy in thyroid gland surgery related to operations and nerves at risk. Arch Surg 120: 475-477, 1985.

14. Scheumann GF, Seeliger H, Musholt TJ, et al. Completion thyroidectomy in 131 patients with differentiated thyroid carcinoma. Eur J Surg Acta Chir 162: 677-684, 1996.

15. Gould EA, Winship T, Philbin PH, et al. Observations on a "sentinel node" in cancer of the parotid. Cancer 13: 7778, 1960.

16. Reintgen D, Cruse CW, Wells K, et al. The orderly progression of melanoma nodal metastases. Ann Surg 220: 759-767, 1994.

17. Miliotes G, Albertini J, Berman C, et al. The tumor biology of melanoma nodal metastases. Am Surg 62: 81-88, 1996.

18. Roh J-L, Koch WM. Role of sentinel lymph node biopsy in thyroid cancer. Expert Rev Anticancer Ther 10: 14291437, 2010.

19. van der Veen H, Hoekstra OS, Paul MA, et al. Gamma probe-guided sentinel node biopsy to select patients with melanoma for lymphadenectomy. Br J Surg 81: 17691770, 1994.

20. Krag DN, Weaver DL, Alex JC, et al. Surgical resection and radiolocalization of the sentinel lymph node in breast cancer using a gamma probe. Surg Oncol 2: 335-339, 1993.
21. Kim JS. Sentinel lymph node biopsy in thyroid cancer. Korean J Endocrine Surg 13: 135-143, 2013.

22. Horvath E, Majis S, Rossi R, et al. An ultrasonogram reporting system for thyroid nodules stratifying sancer risk for clinical management. J Clin Endocrinol Metab 94: 1748-1751, 2009.

23. Davidson HC, Park BJ, Johnson JT. Papillary thyroid cancer: controversies in the management of neck metastasis. The Laryngoscope 118: 2161-2165, 2008.

24. Som PM, Curtin HD, Mancuso AA. An imaging-based classification for the cervical nodes designed as an adjunct to recent clinically based nodal classification. Arch Otolaryngol Head Neck Surg 125: 388-399, 1999.

25. Robbins KT, Medina JE, Wolfe GT, et al. Standardizing neck dissection terminology. Official report of the academy's committee for head and neck surgery and oncology. Arch Otolaryngol Head Neck Surg. 117: 601-605, 1991.

26. Gregoire V, Levendag P, Ang KK, et al. CT-based delineation of lymph node levels and related CTVs in the nodenegative neck: DAHANCA, EORTC, GORTEC, NCIC, RTOG consensus guidelines. Radiother Oncol 56: 135150, 2000.

27. Salvatori GM, Rubello D. Sentinel lymph node biopsy in papillary thyroid cancer. Radio. Surg. 32: 782-785, 2008.

28. Kelemen PR, Van Herle AJ, Giuliano AE. Sentinel lymphadenectomy in thyroid malignant neoplasms. Arch Surg 133: 288-292, 1998.

29. Fukui Y, Yamakawa T, Taniki T, et al. Sentinel lymph node biopsy in patients with papillary thyroid carcinoma. Cancer 92: 2868-2874, 2001.

30. Catarci M, Zaraca F, Angeloni R, et al. Preoperative lymphoscintigraphy and sentinel lymph node biopsy in papillary thyroid cancer. A pilot study. J Surg Oncol 77: 21-24, 2001.

31. Methylene Blue as the safest blue dye for sentinel node mapping: Emphasis on anaphylaxis reaction. PubMed Journals, https://ncbi.nlm.nih.gov/labs/articles/21413854/ (accessed 15.06.2018).

32. Thevarajah S, Huston TL, Simmons RM. A comparison of the adverse reactions associated with isosulfan blue versus methylene blue dye in sentinel lymph node biopsy for breast cancer. Am J Surg 189: 236-239, 2005.

33. Yan DG, Zhang B, Liu L, et al. Sentinel lymph node biopsy in papillary thyroid carcinoma. J Thyroid Disord Ther 3: 140, 2013.

34. Lee SK, Choi JH, Lim HI, et al. Sentinel lymph node biopsy in papillary thyroid cancer: Comparison study of blue dye method and combined radioisotope and blue dye method in papillary thyroid cancer. Eur J Surg Oncol 35: 974-979, 2009. 
International Journal of Hematology and Oncology

35. Yan X, Zeng R, Ma Z, et al. The Utility of Sentinel Lymph Node Biopsy in Papillary Thyroid Carcinoma with Occult Lymph Nodes. PloS One 10(6): e0129304, 2015.

36. Kelemen PR, Van Herle AJ, Giuliano AE. Sentinel lymphadenectomy in thyroid malignant neoplasms. Arch Surg 133: 288-292, 1998.

37. Noguchi S, Murakami N. The value of lymph-node dissection in patients with differentiated thyroid cancer. Surg Clin North Am 67: 251-261, 1987.

38. American Thyroid Association (ATA) Guidelines Taskforce on Thyroid Nodules and Differentiated Thyroid Cancer, Cooper DS, Doherty GM, et al. Revised American Thyroid Association management guidelines for patients with thyroid nodules and differentiated thyroid cancer. Thyroid 19: 1167-1214, 2009.

39. Ito $Y$, Higashiyama $T$, Takamura $Y$, et al. Risk factors for Rrecurrence to the lymph node in papillary thyroid carcinoma patients without preoperatively detectable lateral node metastasis: Validity of prophylactic modified radical neck dissection. World J Surg 31: 2085-2091, 2007.

40. Roh JL, Kim JM, Park Cl. Central cervical nodal metastasis from papillary thyroid microcarcinoma: Pattern and factors predictive of nodal metastasis. Ann Surg Oncol 15: 2482-2486, 2008.
41. Roh JL, Park Cl. Sentinel lymph node biopsy as guidance for central neck dissection in patients with papillary thyroid carcinoma. Cancer 113: 1527-1531, 2008.

42. Schulze T, Bembenek A, Schlag PM. Sentinel lymph node biopsy progress in surgical treatment of cancer. Langenbecks Arch Surg 389: 532-550, 2004.

43. Balasubramanian SP, Harrison BJ. Systematic review and meta-analysis of sentinel node biopsy in thyroid cancer. Br J Surg 98: 334-344, 2011.

\section{Correspondence:}

Le Van QUANG

Hanoi Medical University

Department of Oncology

No: 1 Ton That Tung Street

Dong Da District, HANOI / VIETNAM

Tel: +84 913035195

e-mail: leq89@yahoo.com

lequang@hmu.edu.vn 